\title{
How to Design Internet of Things to Encourage Office Workers to Take More Regular Micro-Breaks
}

\author{
Yitong Huang \\ Mixed Reality Lab \& Horizon Centre for Doctoral Training \\ School of Computer Science, University of Nottingham \\ Nottingham, United Kingdom NG8 1BB \\ +44(0) 1159514251 \\ Yitong.huang@nottingham.ac.uk
}

\begin{abstract}
Prolonged sitting at work has become a new health hazard for office workers. The current $\mathrm{PhD}$ is thus dedicated to exploring the potential of Internet of Things (IoT) for supporting healthier office work and break routines. An "enchanted object" approach that utilizes the "glanceability" and "gesturability" of everyday artefacts is proposed as a potential solution to tackle the challenge of user disturbance and scarcity of cognitive resources in this persuasion context. The vision is to eventually have a collection of digitally "enchanted" office objects that harness ubiquitous sensing and context-aware algorithms to subtly prompt different types of breaks at opportune moments throughout workdays, as a mechanism to break up prolonged sitting; in addition, behavioural data captured from embedded and wearable sensors will be visualized to facilitate self-reflection and habit development. An initial qualitative study is being conducted to unpack challenges and opportunities in reducing prolonged sitting in office work through the lens of both behaviour change and Human Computer Interaction, which has led to preliminary insights to share and discuss with the audience.
\end{abstract}

\section{CCS Concepts}

- Human-centered computing $\rightarrow$ Ubiquitous and mobile computing $\rightarrow$ Ubiquitous and mobile computing theory, concepts and paradigms $\rightarrow$ Ambient intelligence.

\section{Keywords}

Persuasive technology; work health and wellbeing; sedentary behaviours; Internet of Things; environmental persuasion

\section{INTRODUCTION}

Despite a plethora of digital gadgets promoting physical activities, only a few of them are specifically targeting at sedentary behaviours. Recent health research has shown that too much sitting, especially prolonged periods of sitting without breaks, is harmful for metabolic as well as musculoskeletal health [15], regardless of how much exercise one does [11]. A study with 83 office workers highlighted the prevalence of prolonged ( $>55 \mathrm{~min})$ sitting episodes in office work and suggested that sitting reduction interventions

Permission to make digital or hard copies of part or all of this work for personal or classroom use is granted without fee provided that copies are not made or distributed for profit or commercial advantage and that copies bear this notice and the full citation on the first page. Copyrights for thirdparty components of this work must be honored. For all other uses, contact the Owner/Author.

Copyright is held by the owner/author(s).

ECCE '16, September 05-08, 2016, Nottingham, United Kingdom ACM 978-1-4503-4244-5/16/09.

http://dx.doi.org/10.1145/2970930.2970963 should target this population and setting [13]. Whilst unrealistic to entirely eliminate sedentary work from modern society, it is possible to minimize the adverse consequences of occupational sitting, for example, by interspersing sedentary work with microbreaks (3-5 minutes) that involve light activities as simple as walking to the kitchen and refilling a mug, which can alleviate the metabolic dysfunction caused by long periods of sitting [3].

Ubiquitous sensing and context-aware computing have shown much promise for just-in-time and point-of-decision interventions to motivate healthy living [4]. But when it comes to encouraging regular micro-breaks at work, there are two main challenges. Firstly, it is crucial yet difficult to balance the salience and subtlety of the signal sent by the technology, in a way that it delivers the necessary nudge without disturbing or annoying the user who should be engaged in the primary working task. The second challenge is presented by the scarcity of cognitive, especially selfregulatory resources [1] available to someone at work for processing information from persuasive technologies, activating health goals, monitoring own behaviours, and acting in favour of long-term goals.

To address the above two challenges, my $\mathrm{PhD}$ looks beyond existing digital devices and explores the potential of "Enchanted Objects" (EOs) for just-in-time health behaviour change interventions in settings like the office. The term "Enchanted Objects" was first coined by David Rose to describe ordinary everyday objects gaining the magical power from embedded sensors, actuators, wireless transmitters and processors, and interacting with human beings via screen-less "tangible interfaces" to satisfy some fundamental human needs [12]. According to Rose, EOs are characterised by the following three qualities.

Firstly, EOs are glanceable such that they present information at points of need in a readily accessible but nonintrusive manner [12]. In this regard, compared with a popup from the main workstation screen, a break reminder delivered by an enchanted physical object would be a better subtlety-salience compromise.

The second key feature of EOs is gesturability, in a sense that their physical forms suggest actions in line with existing human skills and experience with those everyday objects [12]. For instance, a mug is associated with extensive experiences of tea breaks in office settings; thus an illuminated mug would be naturally perceived as a suggestion for a tea break. This exploits affordances [8] of everyday objects to reduce the cognitive resources required for interpreting messages from persuasive technologies. Moreover, it speaks to the idea of goal activation in cognitive psychology. A goal is a mental representation of a desired end state, the accessibility of which can be enhanced via environmental priming 
[1]. So this presents a possibility that we can make different objects in the office stand out at various time points throughout the working day to activate different goals and prompt different break activities associated with those objects respectively.

The third characteristic of EOs is lovability, in a way that they should support fundamental human needs and provide empowerment. This resonates with Oinas-Kukkonen's suggestion that more persuasive design research should look into building intrinsic motivations and supporting attitude change [10]. So in the current work, apart from individual EOs that enhance selfawareness and send reminders at points of decisions, a standalone multimedia system will be built to feedback data captured from EOs as well as wearable activity trackers to augment self-reflection and support goal-monitoring in the long term.

\section{PAST ACHIEVEMENTS}

In the $1^{\text {st }}$ year of doctoral training, I worked on a collaborative project using wearable cameras to study the nature and context of office work breaks. Two papers have been published so far. The first was a workshop paper titled "Designing for Human Sustainability: the Role of Self-Reflection" (http://dl.acm.org/citation.cfm?id=2794323). It explored the utility of self-reflection both as a research method to elicit design requirements and as a system feature to catalyse learning and behaviour change. I also co-authored a paper, accepted at CHI 2016 and titled "The Role of ICT in Office Work Breaks" (http://dl.acm.org/citation.cfm?id=2858443). The concept of "screen guilt" emerging from this paper has backed my $\mathrm{PhD}$ proposal to explore screen-less interactions with digitally augmented physical objects to deliver work break interventions.

Since Sep. 2015, I have been focusing on two parallel lines of work. I have made a functional prototype of an "omniscient mug" that subtly suggests water breaks at opportune moments based on the user's inactivity time and hydration need. Meanwhile, I have conducted a user study, using a combination of questionnaires, diaries and interviews, to unpack the behaviour change challenge and to inform design. I have collected data from 20 office workers in a variety of job roles, and generated preliminary findings on perceived barriers and facilitators to reduce prolonged sitting at work and on the potential acceptability of EOs as a mode of delivering work health interventions.

\section{FUTURE DIRECTION AND POTENTIAL CONTRIBUTIONS}

My next step is a more systematic analysis of the interview data I have collected, using the Theoretical Domain Framework (TDF) [2], which is an integrative framework that groups theories of behaviour change with overlapping constructs into 14 theoretical domains, including social and environmental aspects as well as common psychological constructs. By applying TDF to coding the data, I will be able to identify all possible facets that influence break-taking behaviours, and disentangle the question of what really needs to be changed for the desired behaviour change to occur.

This further analysis will both feed into later $\mathrm{PhD}$ work and inform other ergonomists' and health scientists' work on reducing prolonged sitting in office settings. Actually, interventions about micro-breaks in Visual Display Terminal (VDT) work have featured in ergonomics literature for several decades, usually in relation to boosting productivity or/and reducing syndromes associated with screen use [14]. Undoubtedly, the success of such interventions is reliant on the user changing their behaviour. However, it is a shame that studies in this domain have rarely framed or addressed the issue as a behaviour change challenge. So a qualitative study that unpacks the behavioural and psychological determinants to prolonged sitting will be very informative.

Ultimately, I would like to design, prototype and evaluate a digital intervention informed by theoretical analysis and empirical evidence in my $\mathrm{PhD}$. To achieve that, I will hybridize the usercentric approach to designing socio-technical systems from HCI [5] with evidence-based practice for designing theory-based behaviour change interventions from behavioural medicine [7]. For the intervention design, I will draw on a set of tools to help me link the behavioural diagnosis to appropriate choice of intervention functions [7], behaviour change techniques (BCTs) [6] and mode of delivery (MoD) [16]. However, as EOs or even IoT in general is still a rather novel MoD for behaviour change, not even found in the most current technology coding scheme [16], presenting a dearth of high quality intervention studies with this MOD to back up its value. So a second potential contribution from my $\mathrm{PhD}$ will be an insight into the feasibility, utility and acceptability of using EOs for delivering behaviour change interventions, especially in the context of office work health.

Finally, as my user study sheds light on the importance of automatic, unconscious and habitual processes in determining work break behaviours, I have come to propose a framework for conceptualising potential roles EOs can play in nurturing health habits "in the wild". Therefore, I am planning to incorporate some experimental paradigms from social cognition in my evaluation study. For instance, a lab-based procedure including priming, lexical decision and Stroop measures of goal accessibility, and/or implicit affective association tests [9] can be administered to participants before and after they use the mug-based break reminders for an extended period of time in real life. In this way, we may be able to measure underlying constructs changed by EOs (e.g. object-primed goal accessibility, affect associated with breaks) and trace the causal pathways mediating EOs' effects on behaviours. This will consequently contribute to the "habit" literature with theories and evidence for the utility of IoT in studying and changing everyday habits in real-life settings.

\section{ISSUES EXPERIENCED AND ADVICE SOUGHT}

First, as the part relating to HCI research questions in my user study is not as structured as that for behavioural change, it is hard to extract useful insights from this dataset. Therefore, I am looking for analytic frameworks to query the data through the lens of system engineering. Another issue pertains to elicitation of user requirements and inspirations for futuristic digital designs like EOs. I asked participants to record in diaries "when...(context), I would like my...(object) to say to me...(message)" with the hope to gather some inspirations. However, responses to this question have been quite sporadic and of varying quality. So I am in need of advice on more fruitful methods for engaging potential users in design and research. Finally, I would like to solicit feedback from a cognitive engineering perspective on my overall $\mathrm{PhD}$ proposal of EOs as well as on specific design ideas, such as impactful ways of visualising sensor data to facilitate users' self-reflection.

\section{ACKNOWLEDGEMENTS}

This author is supported by the Horizon Centre for Doctoral Training at the University of Nottingham (RCUK Grant No. EP/L015463/1), the RCUK's Horizon Digital Economy Research Institute (RCUK Grant No. EP/G065802/1) and Unilever UK Ltd. 


\section{REFERENCES}

[1] Baumeister, R.F., Bratslavsky, E., Muraven, M. and Tice, D.M. 1998. Ego depletion: is the active self a limited resource? Journal of personality and social psychology. 74, 5 (1998), 1252-1265.

[2] French, S.D., Green, S.E., O’Connor, D.A., McKenzie, J.E., Francis, J.J., Michie, S., Buchbinder, R., Schattner, P., Spike, N. and Grimshaw, J.M. 2012. Developing theory-informed behaviour change interventions to implement evidence into practice: a systematic approach using the Theoretical Domains Framework. Implementation science : IS. 7, 1 (Jan. 2012), 38.

[3] Healy, G.N., Dunstan, D.W., Salmon, J., Cerin, E., Shaw, J.E., Zimmet, P.Z. and Owen, N. 2008. Breaks in sedentary time: beneficial associations with metabolic risk. Diabetes care. 31, 4 (Apr. 2008), 661-666.

[4] Intille, S. 2002. Designing a home of the future. IEEE Pervasive Computing. 1, (2002), 76-82.

[5] Maguire, M. 2001. Methods to support human-centred design. International Journal of Human-Computer Studies. 55, (2001), 587-634.

[6] Michie, S., Richardson, M., Johnston, M., Abraham, C., Francis, J., Hardeman, W., Eccles, M.P., Cane, J. and Wood, C.E. 2013. The behavior change technique taxonomy (v1) of 93 hierarchically clustered techniques: Building an international consensus for the reporting of behavior change interventions. Annals of Behavioral Medicine. 46, 1 (2013), 81-95.

[7] Michie, S., van Stralen, M.M. and West, R. 2011. The behaviour change wheel: A new method for characterising and designing behaviour change interventions. Implementation science: IS. 6, 1 (2011), 42.

[8] Norman, D. 1988. The Psychology of Everyday Things. Basic Books.
[9] Nosek, B. a, Hawkins, C.B. and Frazier, R.S. 2011. Implicit social cognition: from measures to mechanisms. Trends in cognitive sciences. 15, 4 (Apr. 2011), 152-9.

[10] Oinas-Kukkonen, H. 2010. Behavior change support systems: A research model and agenda. Proceedings of the 5th International Conference, PERSUASIVE 2010. (2010), 4-14.

[11] Pate, R.R., O’Neill, J.R. and Lobelo, F. 2008. The evolving definition of "sedentary". Exercise and sport sciences reviews. 36, 4 (2008), 173-178.

[12] Rose, D. 2014. Enchanted Objects: Design, Human Desire, and the Internet of Things. Simon and Schuster.

[13] Ryan, C.G., Dall, P.M., Granat, M.H. and Grant, P.M. 2011. Sitting patterns at work: objective measurement of adherence to current recommendations. Ergonomics. 54, 6 (2011), 531538.

[14] Swanson, N.G. and Sauter, S.L. 1990. The design of rest breaks for video display terminal work: A review of the relavant literature. Journal of Safety Research. 21, (1990), 166.

[15] Tremblay, M.S., Colley, R.C., Saunders, T.J., Healy, G.N. and Owen, N. 2010. Physiological and health implications of a sedentary lifestyle. Applied Physiology, Nutrition, and Metabolism. 35, 6 (2010), 725-740.

[16] Webb, T.L., Joseph, J., Yardley, L. and Michie, S. 2010. Using the internet to promote health behavior change: a systematic review and meta-analysis of the impact of theoretical basis, use of behavior change techniques, and mode of delivery on efficacy. Journal of medical Internet research. 12, 1 (Jan. 2010), 1-24. 